\title{
Thermal Lensing Spectroscopy with Picosecond Pulse Trains and a New Dual Beam Configuration
}

\author{
J. W. PERRY, E. A. RYABOV ${ }^{a}$ and A. H. ZEWAIL ${ }^{b}$
}

Arthur Amos Noyes Laboratory of Chemical Physics, ${ }^{\circ}$ California Institute of Technology, Pasadena, California 91125 USA

(Received May 17, 1982)

It is now well-known that thermal lensing (TL $)^{1,2}$ induced by CW or pulsed lasers can be used for spectroscopic study of weak transitions, ${ }^{3}$ for trace analysis, ${ }^{4}$ for investigation of chemical kinetics ${ }^{4}$ and for nonlinear spectroscopy. ${ }^{5,6}$ TL spectrometers employing single and dual (pump, probe) beam methods have been reported. ${ }^{3}$ The time dependence of the thermal lensing signal is determined by the rate of thermalization of the deposited energy, the velocity of sound in the material, and the rate of thermal conduction. The rate of thermalization (with time constant $\tau$ ), may depend on the lifetime of the excited state (population depletion), while the time constant for dissipation of the thermal lens is given ${ }^{1}$ by $t_{\mathrm{c}}=w^{2} \rho C_{\mathrm{p}} / 4 k$, where $w$ is the laser beam diameter, $\rho$ is the density of the material, and $C_{\mathrm{p}}$ and $k$ are the specific heat and thermal conductivity, respectively. The thermalization time constant $(\tau)$ is typically short (microsecond timescale) ${ }^{7,8}$ In contrast, $t_{\mathrm{c}}$ is usually long, on the order of milliseconds. ${ }^{2}$

\footnotetext{
${ }^{a}$ Visiting Research Associate. Permanent address: Institute of Spectroscopy, USSR Academy of Sciences, 142092 Moscow Region, Troitzk, USSR.

${ }^{b}$ Alfred P. Sloan Foundation Fellow and Camille \& Henry Dreyfus Foundation TeacherScholar.

'Contribution No. 6648
} 
In this communication, we wish to report on the use of synchronously mode-locked picosecond lasers in a pump-probe configuration for TL spectroscopy. The peak power for these picosecond lasers is very high and, of course, the fundamental of the dye laser (red beam) can be efficiently $(\sim 10 \%)$ doubled in frequency (U.V. beam) by second harmonic generation in nonlinear crystals. We use this generated U.V. beam as a probe to monitor the very weak absorption of the red beam. An arrangement ${ }^{4}$ of the beams involving different waist positions for the pump and probe is used. This arrangement results in an enhancement of sensitivity (at least a factor of three to seven). Also, a different dependence of the signal on the cell position compared to the single beam method is obtained. Finally, we have obtained the $\Delta v=5 \mathrm{CH}$-stretching overtone absorption spectrum of liquid toluene with this method (see Figure 1). Suggestions are made regarding new applications of this picosecond pulse thermal lensing technique.
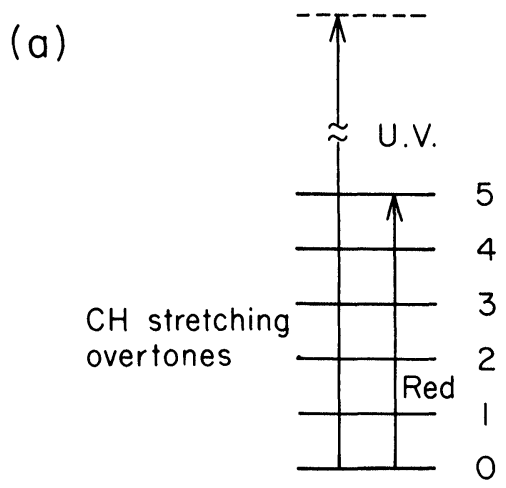

(b)

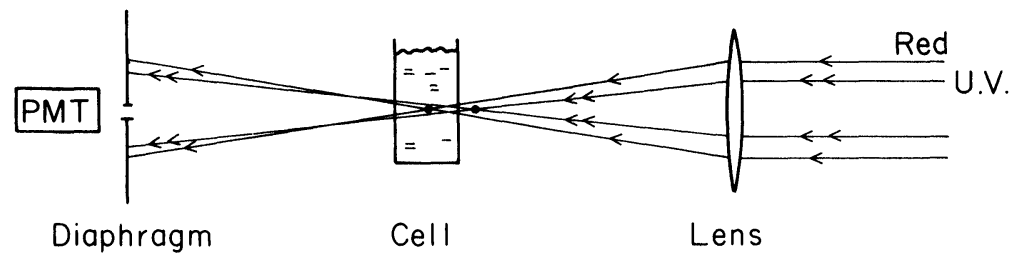

FIGURE 1 Schematic of energy levels (upper) and experimental geometry (lower). 
A mode-locked argon ion laser (Spectra Physics 171) (frequency $\sim 82$ $\mathrm{MHz}$ ), which produces a $0.9 \mathrm{~W}$ pulse train at $5145 \AA$ with pulse widths of $\sim 100 \mathrm{ps}$, was used to pump a dye laser. The dye laser gain medium ${ }^{9}$ was 1:1 mixture of DCM and LD700 $(1.2 \mathrm{M})$ dissolved in $25 \%$ benzyl alcohol, 25\% glycerol and 50\% dimethyl sulfoxide. This dye laser operates over the range of $705-770 \mathrm{~nm}$ and produces $\sim 100 \mathrm{~mW}$ at the peak, with an $8 \%$ output coupler. The dye laser had a pulse width of $<10$ ps. A 1.5 mm thick $\mathrm{LiIO}_{3}$ crystal, cut for phase matching at $720 \mathrm{~nm}$, was used to frequency double the dye laser output. The beams were focused, in a colinear geometry, into the cell with toluene using a $75 \mathrm{~mm}$ focal length lens. The fundamental power at the sample was $\sim 50 \mathrm{~mW}$ and U.V. power was $\sim 30-50$ times lower. Heating of the sample due to absorption of the U.V. beam was negligible.

Using a telescope to adjust the U.V. beam, separated from the red by a dichroic mirror, allowed variation of the relative position of the beam waists. The quartz cell $(1$ or $10 \mathrm{~mm})$ filled with toluene was translated along the beam axis. The intensity of the red or U.V. beam, transmitted through a $1 \mathrm{~mm}$ diameter $(\varphi)$ diaphragm (placed $\sim 120 \mathrm{~mm}$ from the red beam waist position) was measured in the experiments on the cell position dependence of the signal (Figure 1).

Figure 2a shows the observed dependence ${ }^{10}$ of the red beam intensity on cell position for the single beam experiment with a $1 \mathrm{~mm}$ cell. The shape of this curve is in good agreement with that predicted by theory. ${ }^{1}$ The maximum signal $\left(S=I_{0}-I / I_{0}\right)$ achieved in this experiment was 9.4\%. In the double beam experiment, with mismatched focusing, the intensity of the UV beam had a different dependence on the cell position. In these experiments, the UV beam waist position occurs before the red beam waist position. Thus, the cell position dependence of the transmitted intensity shows no change in sign, Figure $2 \mathrm{~b}$. We find only a decrease in the UV intensity as the cell approaches the red beam waist position, as expected. The maximum signal for the dual beam experiment was $30 \%$ at the red beam waist position, showing a much larger value than for the single beam experiment. In fact, a "black-hole" appeared visible to the naked eye on the UV beam!

For the long cell $(l=10 \mathrm{~mm})$ the position dependence of the red beam intensity is more complicated than for the thin $(1 \mathrm{~mm})$ cell, Figure 3a. In this case $l \gg z$ where $z=\pi w_{0}^{2} / \lambda(\mathrm{z} \sim 1 \mathrm{~mm})$, the confocal length parameter. Thus, the thermal lens cannot be considered as thin. This is probably why there exists a "plateau" between the maximum and minimum. For the dual 


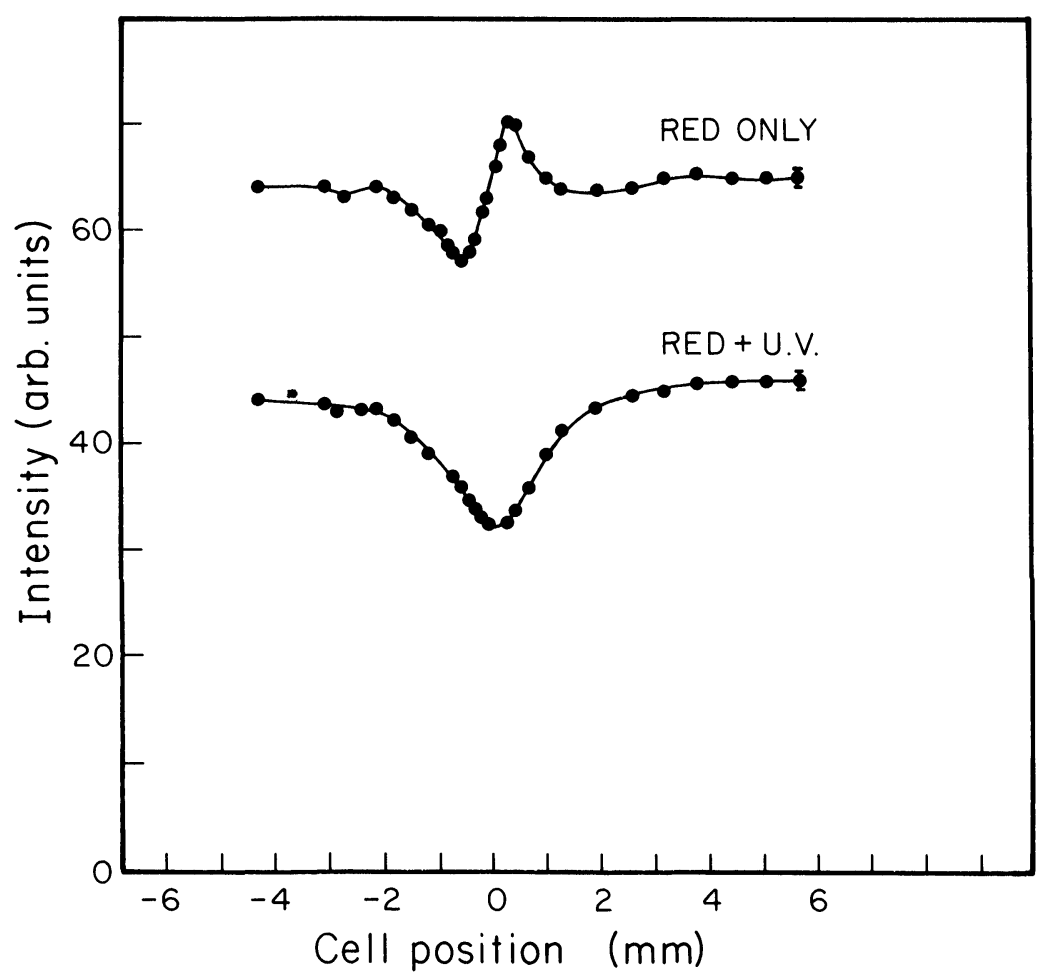

FIGURE 2 Observed cell position dependence of transmitted probe beam intensity for the $1 \mathrm{~mm}$ thick cell with toluene liquid, $\lambda$ exc. $=715 \mathrm{~nm}$. a) upper curve is with red beam only and b) lower curve is for the dual beam method (red and UV beams) and the UV intensity is plotted.

beam experiment with the thick cell the intensity follows roughly the same dependence on cell position as for the thin cell, except for being broader, close to the cell length. Again, the maximum signal for the thick cell for the dual beam case is more than six times the signal in the single beam case. With these results, we have demonstrated experimentally that an increased signal (larger defocusing effect) results when a dual beam method with mismatched focusing ${ }^{4}$ from a short focal length lens is used, relative to the single beam case, for the thermal lensing. ${ }^{11}$

Using the new dual beam configuration we have obtained the $\Delta v=5$ $\mathrm{CH}$-stretching overtone spectrum of liquid toluene, Figure 4 . This spectrum agrees reasonably well with the spectrum measured with a conventional 


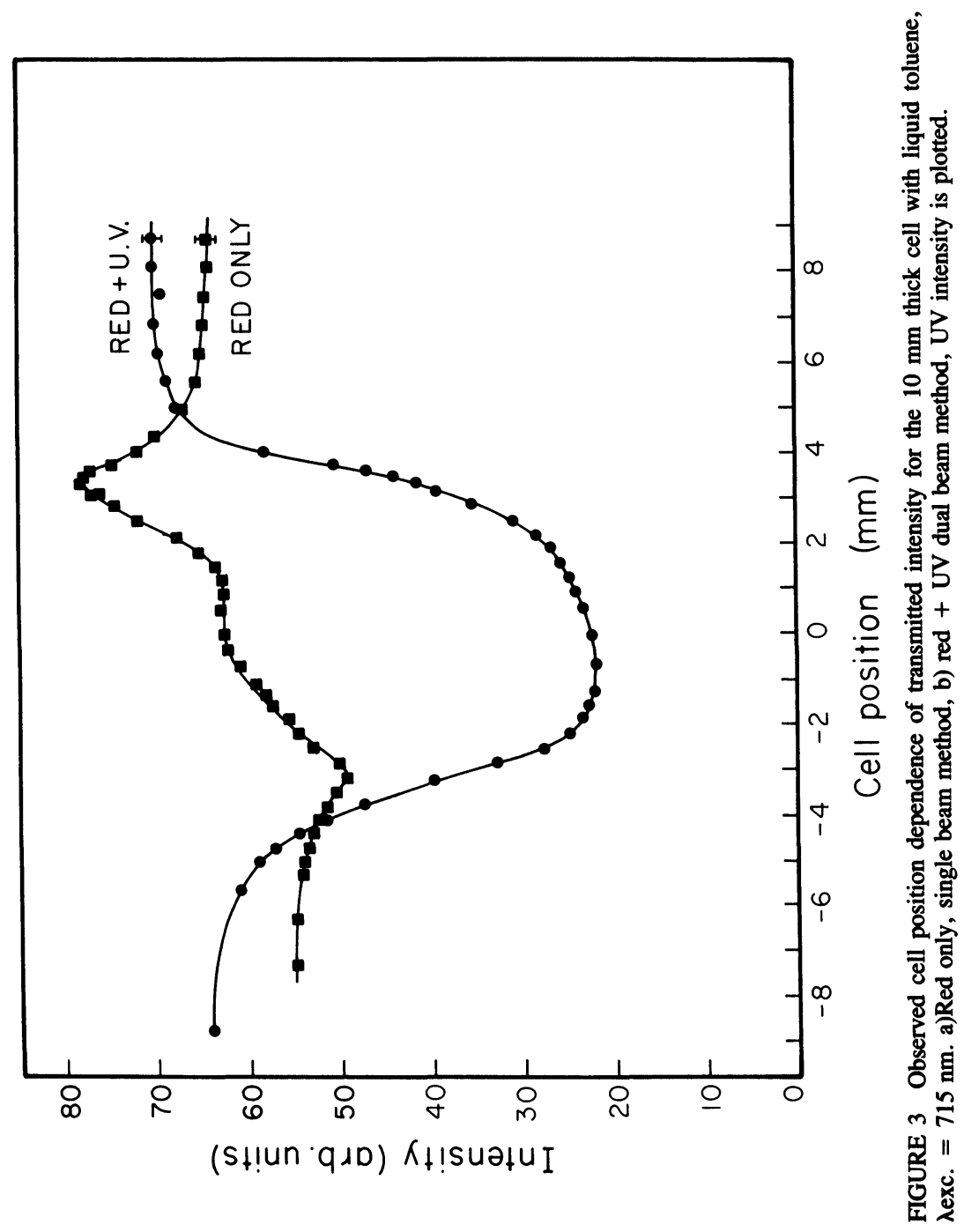


spectrophotometer and using a $10 \mathrm{~cm}$ path length. Thus, the picosecond pulse thermal lensing spectrometer gives reasonable linear absorption spectra, as is confirmed again by the absence of absorption by benzene- $d_{6}$ in this spectral region. In contrast with benzene- $d_{6}$, the "black hole" was seen in benzene- $h_{6}(\Delta v=5)$, and toluene $(\Delta v=6)$.

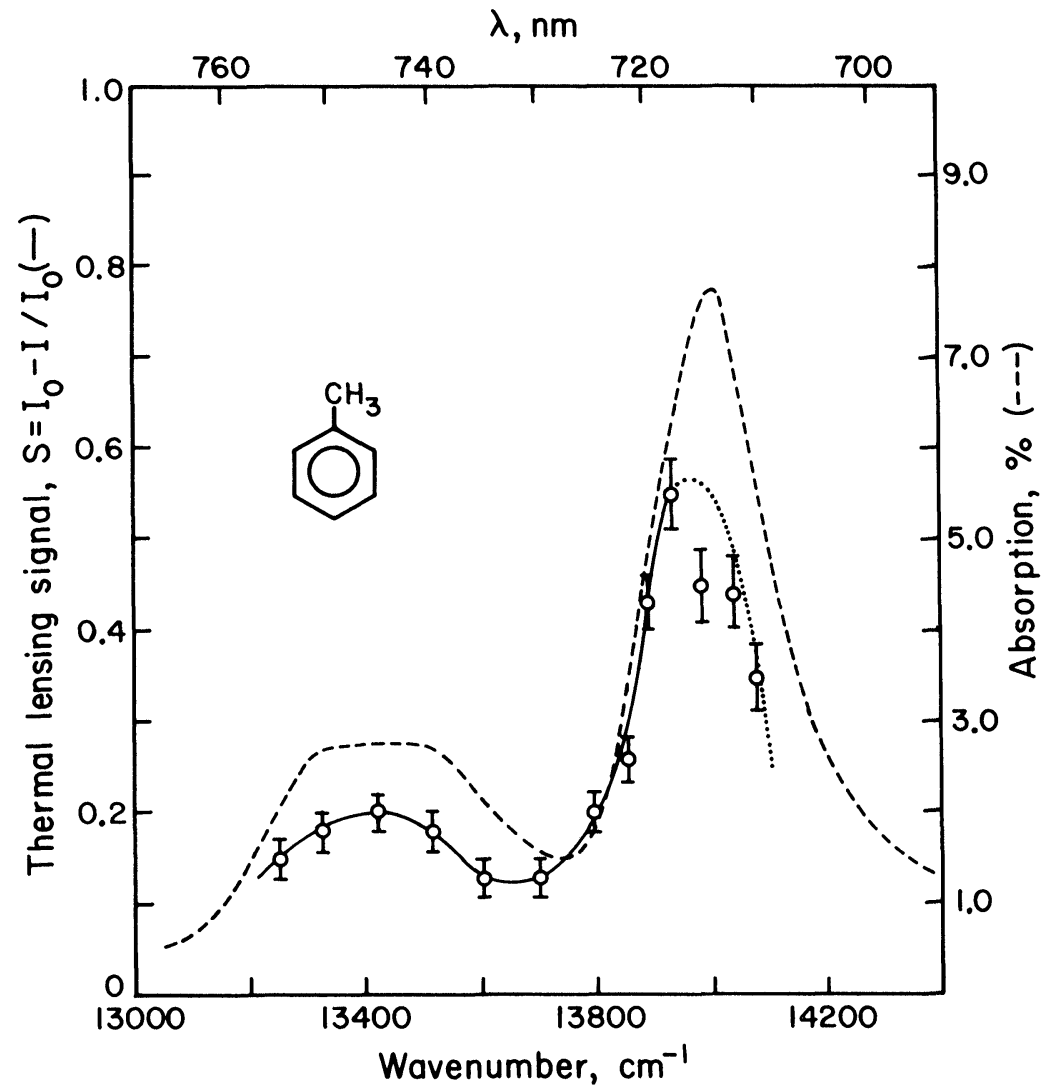

FIGURE $4 \mathrm{CH}$ stretching overtone spectrum $\left(\Delta v_{\mathrm{CH}}=5\right)$ of liquid toluene at room temperature $\left(23^{\circ} \mathrm{C}\right)$. (----) spectrum obtained with $10 \mathrm{~cm}$ cell path length and Cary-17 Spectrophotometer. ( - , with error bars) spectrum obtained with dual-beam picosecond thermal lensing spectrometer. The absorption scale is defined as $100 \%$ minus the transmission. It should be mentioned that the peak around $13400 \mathrm{~cm}^{-1}$ exhibits clear structure in the gas phase $^{12}$; however, broadening of the bands in the liquid results in near coalescence, thus giving the unusual bandshape. Also, the conditions under which the TL spectrum was obtained were far from optimum. However, the spectrum suffices for the purpose of showing that we are dealing with linear overtone absorption. The spectra agree with that obtained previously. ${ }^{13}$ 
New applications of this method can be anticipated, such as the measurement of nonlinear absorption (e.g., two-photon absorption) in liquids and gases. These measurements should be successful because of the possibility of high peak power from picosecond pulses. Using these lasers with the appropriate repetition rate and delays between the pump and probe pulses, a means for monitoring the risetime of nonthermal transient lensing ${ }^{6}$ (e.g., plasma formation) should be obtained.

\section{Acknowledgment}

This work was supported by grants from the National Science Foundation (CHE8112833 and DMR8105034). We would like to thank Professor D. Kliger for his useful comments.

\section{References}

1. J. P. Gordon, R. C. C. Leite, R. S. Moore, S. P. S. Porto, and J. R. Whinnery, J. Appl. Phys. 36, 3 (1965).

2. C. Hu and J. R. Whinnery, Appl. Optics 12, 72 (1973).

3. R. L. Swofford, M. E. Long, and A. C. Albrecht, J. Chem. Phys. 65, 179 (1976).

4. J. P. Haushalter and M. D. Morris, Appl. Spectrosc. 34, 445 (1980).

5. D. S. Kliger, Acc. Chem. Res. 13, 129 (1980).

6. G. C. Nieman and S. D. Colson, J. Chem. Phys. 68, 2994 (1978).

7. F. W. Dabby, R. W. Boyko, C. V. Shank, and J. R. Whinnery, IEEE J. Quantum Electron. QE-5, 516 (1969).

8. D. R. Siebert, F. R. Grabiner and G. W. Flynn, J. Chem. Phys. 60, 1564 (1974).

9. E. Marason and R. Boggy (Spectra Physics)-private communication.

10. Note that the maximum signal for the single beam case occurs at $\sim 0.5 \mathrm{~mm}(\sim$ one confocal length) from the zero cell position (red beam waist) in agreement with prediction. ${ }^{1}$

11. This observation may be physically justified in that the temperature gradient generated from the pump beam is large (with a short focal length lens) and the resulting thermal lens is located near one confocal length, the maximum defocusing position, ${ }^{2}$ from the probe beam waist.

12. K. V. Reddy, D. Heller, and M. Berry, J. Chem. Phys. 76, 2814 (1982).

13. M. S. Burberry, J. A. Morrell, A. C. Albrecht, and R. L. Swofford, J. Chem. Phys. 70, 5522 (1979). 